\title{
Anecdotes from the pandemic
}

\author{
Rapid transformation of the research landscape as a result of COVID-19 and the response to this pandemic has \\ resulted in unexpected challenges
}

T: he second anniversary of COVID19 being declared a pandemic by the World Health Organization is approaching. As we are reminded by daily pandemic statistics, many lives have been lost during this public health emergency. Somewhat less quantifiable is the effect on livelihoods, including those of academic immunology researchers. Painting a picture of how the pandemic has affected immunology research and researchers is rather difficult - with labs being retooled to fight SARS-CoV-2, fluctuating supplies of vaccines, the emergence of variants of concern and polarized governmental and funding responses to the pandemic within and among countries (see our Coping with COVID series) - but we think it is important to try.

Only a few months into the pandemic, Nature Human Behaviour published a Comment based on a survey of principal investigators. The study showed that women, those with children and 'bench' researchers had suffered the greatest hit to the number of hours that they could spend on research each week as many countries and institutions imposed mandatory lockdowns. The least affected disciplines were mathematics, statistics and computer sciences, whereas the most affected was 'Biochemistry, cellular \& molecular biology'. Although we here at Nature Immunology have not conducted such a survey to see how much has changed since those early days, we did contact several researchers around the globe for their perspectives. The following information is a priori anecdotal evidence from a limited number of responses and therefore should not be considered as globally representative or specific to any one institution or country.

In 2020, the world mobilized to fight SARS-CoV-2 and many researchers told us that they were grateful for the extra funding that was made available to enable research programs to be extended or to switch to COVID-19 research. The automatic extension of $\mathrm{PhD}$ programs was a common theme. Institutional support also enabled the rapid downsizing of mouse colonies without the loss of rare or valuable mouse lines before the mass closure of many laboratories - although we also heard that in at least one instance, researchers were not notified soon enough that colonies would be culled by their institution. Among the many other difficulties that have affected immunology researchers over the past two years, staff absences through illness, workplace closures, childcare issues, travel bans and a lack of basic safety and laboratory kits were reported as key issues for some. A shortage of filtered pipette tips in the United States and a global shortage of non-human primates also occurred, both of which were sequestered, for COVID-19 testing and research, respectively.

Among those researchers who were able to keep labs running early in the pandemic, many turned their attention to COVID-19, resulting in a diversity of expert contributions to research in this field. Some COVID-19 grant programs were overwhelmed, resulting in very low rates of success. Furthermore, owing to the rapid rate of discovery in this field, some grant proposals were outdated even before the funding panels were able to assess them. Several sources also stated that (in the United States and Europe at least) COVID-19 funding quickly dried up as a result of the success of the vaccine programs. Perceptions were that much of the new COVID-19 funding seems to now be directed towards research into long COVID-19. However, one researcher noted that the term 'long COVID$19^{\prime}$ is not yet clearly defined, which makes it hard to determine what type of research is eligible for funding under these programs.

Another researcher was concerned that these funding patterns might result in a loss of focus on the virus and pointed to the SARS outbreak in 2003, which quickly lost international attention once the immediate danger was averted - the implication being that if we had learned the global health lessons of today back then we might not be in the predicament we are now.

Another perception among some researchers is that academic journals are less interested in - or have less space for - non-COVID-19 research papers. Here at Nature Immunology, we have not restricted our pages to COVID-19 studies. We strive to publish solid immunology in all areas of broad interest. COVID-19 papers are assessed and reviewed based on the same criteria of novelty and importance as any other paper in an emerging field of high interest. Once accepted, COVID-19 papers are fast-tracked during production, as per our policy across the Nature journals. Some authors reported frustrating delays during peer review and publication of non-COVID-19 content. Like everybody else, reviewers have found themselves swamped trying to juggle their labs, personal lives and health during the pandemic. At Nature Immunology, we have tried to accommodate both referees and authors and have seen only a slight delay in manuscript turnaround times, despite a huge spike in submission rates since the beginning of the pandemic.

The collaboration and speed of discovery in the COVID-19 field has been inspirational, and the success of vaccination programs can be considered one of the great achievements of modern science. There is also now a hope that many of the lessons learned during this time will benefit us in the future, with a better understanding and emphasis on vaccine research, clinical immunology and public health and a preparedness for future infectious outbreaks. Nevertheless, as our unofficial survey revealed, we need to be continuously aware of the challenges and difficulties faced by immunology researchers at all levels.

Published online: 23 September 2021 https://doi.org/10.1038/s41590-021-01039-4 